\title{
Disección coronaria espontánea: ¿revascularización o manejo conservador?
}

\section{Spontaneous coronary artery dissection: Revascularization or conservative management?}

Elizabet Méndez-Eirín*, José Á. Rodríguez-Fernández, Borja Souto-Caínzos, Yago Suárez-Ouréns, Ramón Calviño-Santos y José M. Vázquez-Rodríguez

Servicio de Cardiología, Complejo Hospitalario Universitario A Coruña, Instituto de Investigación Biomédica de A Coruña (INIBIC), A Coruña, España

La disección coronaria espontánea (DCE) es una causa infrecuente de síndrome coronario agudo (SCA) que afecta predominantemente a mujeres jóvenes sin apenas factores de riesgo cardiovascular ${ }^{1}$. Se desconoce la verdadera prevalencia de la enfermedad, dado que es una entidad infradiagnosticada. Según publicaciones recientes, la DCE se diagnosticó mediante coronariografía en el $0.1-4 \%$ de todos los $\mathrm{SCA}^{2}$. A diferencia del SCA secundario a placa de ateroma complicada, donde se ha confirmado que el tratamiento de elección es la revascularización precoz, en los pacientes con eventos coronarios agudos en relación con DCE, no hay evidencia sobre cuál es la mejor estrategia de tratamiento, por lo que las decisiones terapéuticas continúan siendo un reto.

Presentamos dos casos de SCA en relación con DCE en los que el manejo terapéutico ha sido diferente.

El primer caso es el de una paciente de 40 años, sana, con un cuadro de angina de una hora de evolución, objetivando en el electrocardiograma un descenso generalizado del segmento ST y ascenso del ST de $1 \mathrm{~mm}$ en $\mathrm{V} 1 \mathrm{y}$ aVL, que se normaliza tras desaparecer la clínica tras la administración de nitroglicerina sublingual. Un ecocardiograma mostró disfunción sistólica ligera a expensas de hipocinesia anterolateral y anterior. Se realizó una coronariografía preferente que detectó una lesión en tronco común izquierdo compatible con DCE tipo 1, decidiendo manejo conservador. En las siguientes 24 horas presentó recurrencia de angina, el electrocardiograma mostraba datos de isquemia extensa y un ecocardiograma evidenció disfunción sistólica moderada, por lo que se realizó un cateterismo emergente en el que se detectó además de la lesión del tronco común izquierdo presente en el estudio previo, una oclusión del ostium de la circunfleja (Fig. 1A), por lo que se realizó intervencionismo coronario percutáneo (ICP) con implante de stents farmacoactivos sobre tronco común y circunfleja proximal (Fig. 1B). Un ecocardiograma de control previo al alta hospitalaria mostró una fracción de eyección del ventrículo izquierdo del $35 \%$ a expensas de acinesia anterolateral, anterior y septal anterior e hipocinesia inferolateral, y el pico de troponina I fue de $14,095 \mathrm{ng} / \mathrm{l}$. La paciente se mantuvo clínicamente estable tras la revascularización y el tratamiento al alta incluyó doble antiagregación (ácido acetilsalicílico y ticagrelor), un betabloqueante, un inhibidor de la enzima convertidora de angiotensina y un antagonista de la aldosterona. Durante la evolución, en los cinco meses posteriores, la paciente ha 


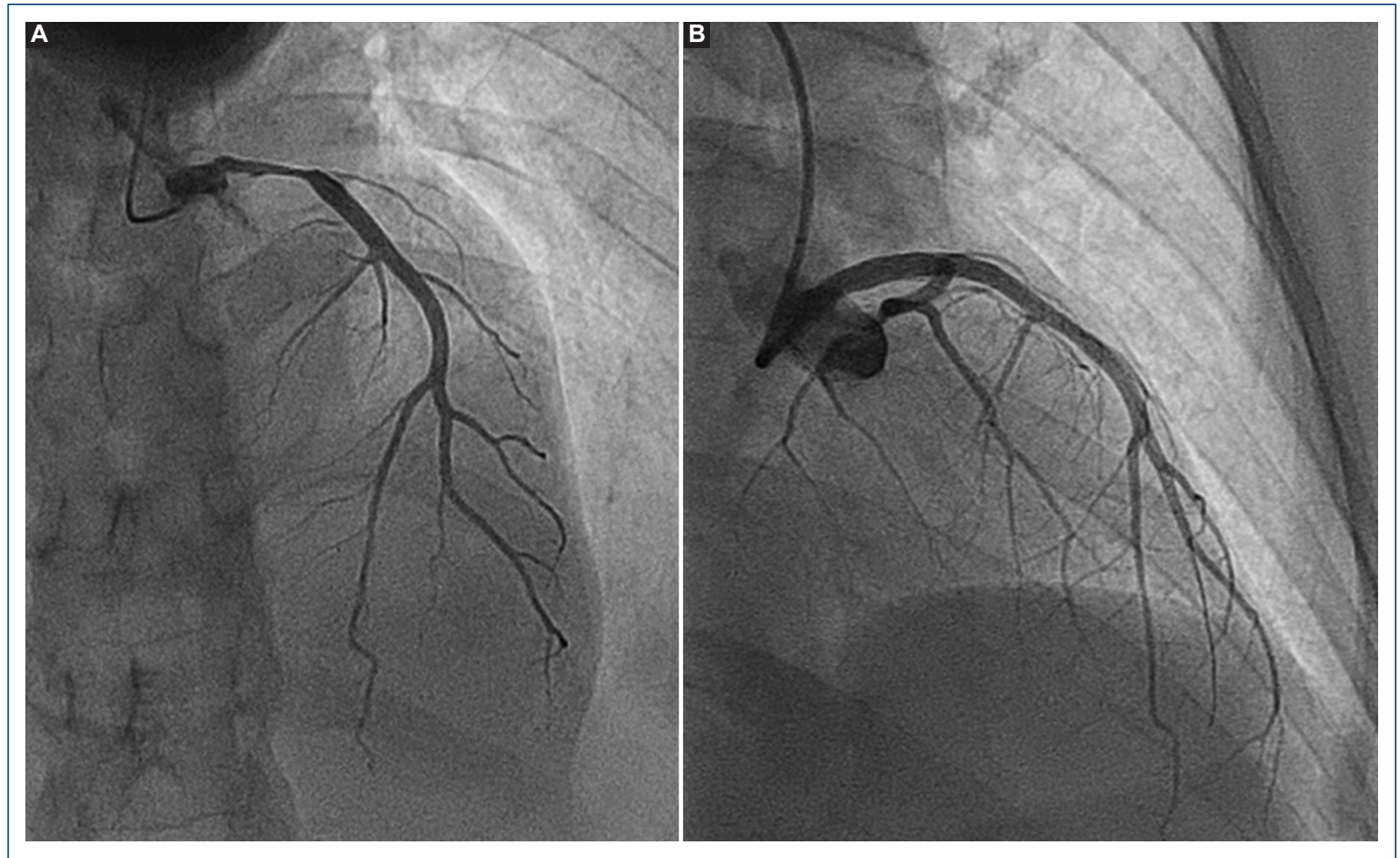

Figura 1. A: coronariografía que muestra disección coronaria espontánea del tronco común izquierdo y oclusión ostial de la arteria circunfleja. B: resultado tras el intervencionismo coronario percutáneo.

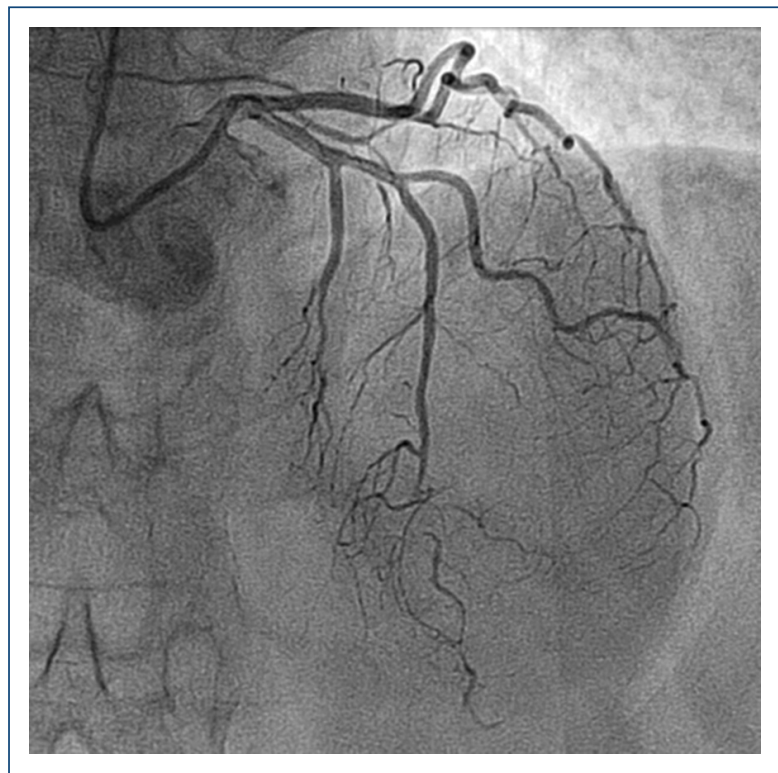

Figura 2. Coronariografía que muestra disección coronaria espontánea a nivel del segmento distal de la arteria descendente anterior y de la primera rama diagonal. permanecido estable. Un ecocardiograma confirmó normalización de la función sistólica ventricular izquierda, y una angiografía por resonancia magnética cerebral y por tomografía computarizada de aorta torácica, abdominal y del eje iliofemoral, descartaron la presencia de aneurismas o signos de displasia fibromuscular (DFM).

El segundo caso es el de una mujer de 60 años con antecedente de hipertensión arterial, por lo que tomaba un antagonista del receptor de la angiotensina II, y una tiroiditis crónica autoinmune, que consulta por un cuadro de angina de dos horas de evolución, detectando en el electrocardiograma a su llegada al hospital datos de isquemia subepicárdica anterolateral. Tras el inicio de nitroglicerina endovenosa cedió la sintomatología. Se realizó una coronariografía preferente en la que se detectaron dos lesiones severas a nivel del segmento distal de la descendente anterior y en la primera rama diagonal, compatibles con DCE tipo 2 (Fig. 2), que se manejaron de forma conservadora. El pico de 
troponina I fue de 13,140 ng/l y un ecocardiograma mostró función sistólica global conservada e hipocinesia apical y septal media. La evolución posterior resultó satisfactoria, sin recurrencia de angina, y el tratamiento al alta incluyó ácido acetilsalicílico y un betabloqueante, además de mantener el tratamiento antihipertensivo que tomaba. La evolución en los 12 meses posteriores ha sido favorable, y se ha descartado la presencia de DFM mediante técnicas de imagen vascular.

Para el diagnóstico de DCE, la coronariografía, utilizando técnicas de imagen intracoronaria, es la prueba gold standard. La imagen angiográfica típica de DCE consiste en una doble luz con retención de contraste extraluminal, junto a múltiples defectos de repleción, configurándole en ocasiones una apariencia de disección espiroidea. Sin embargo, cuando los hallazgos angiográficos no son patognomónicos, la DCE puede pasar inadvertida. En el año 2014, Saw et al. publicaron un trabajo en el que describen tres variantes de DCE, basándose en las características angiográficas ${ }^{3}$ : tipo 1 , imagen clásica de doble lumen lineal con identificación de un flap radiolucente, con frecuencia asociado a retención de contraste en la falsa luz; tipo 2, en la que se detecta una reducción difusa del calibre arterial; y tipo 3 , en la que se visualiza una estenosis focal o tubular (normalmente $<20 \mathrm{~mm}$ ), prácticamente indistinguible mediante angiografía de la estenosis secundaria a aterosclerosis. Se recomienda el uso de técnicas de imagen intracoronaria como la ecografía intravascular o la tomografía de coherencia óptica para confirmar el diagnóstico de DCE.

Las recomendaciones actuales de manejo de los pacientes con DCE se basan en estudios observacionales y opiniones de expertos, dado que no se dispone de ensayos clínicos aleatorizados. En general, se considera que el manejo conservador es la estrategia terapéutica de elección, dado que en el $70-97 \%$ de los casos la evolución es favorable y la arteria se repara por completo de forma espontánea ${ }^{4,5}$. Además, el ICP se asocia con elevadas tasas de complicaciones y resultados frecuentemente subóptimos ${ }^{2}$, incluyendo el riesgo de disección iatrogénica y extensión de la disección o hematoma proximal o distal al stent implantado, lo que puede llevar a la pérdida de flujo ${ }^{6}$. Por otra parte, como se trata habitualmente de lesiones largas, se requiere con frecuencia el empleo de stents de mayor longitud, lo que aumenta el riesgo de reestenosis y trombosis en el seguimiento.

La revascularización percutánea debe reservarse para pacientes que presenten características de alto riesgo, como evidencia clínica de isquemia en curso (angina recurrente, cambios electrocardiográficos), shock cardiogénico, arritmias ventriculares o disección del tronco coronario izquierdo con anatomía técnicamente favorable para ICP $\mathrm{ICn}^{7}$. Equellos casos en los que el ICP resulte fallido o se considere de muy alto riesgo, se debe considerar la cirugía de revascularización coronaria ${ }^{8}$. No obstante, los resultados de la revascularización quirúrgica no son generalmente buenos, debido a que la pared de la arteria coronaria disecada es muy frágil, siendo frecuentes las complicaciones a nivel de las anastomosis.

En cuanto al tratamiento médico en estos pacientes, se recomienda el uso de ácido acetilsalicílico, pero existe controversia respecto a la indicación de doble terapia antiagregante si no se realiza revascularización percutánea ${ }^{4,9}$. Está recomendado el uso de betabloqueantes, ya que son fármacos que disminuyen el estrés parietal y reducen el riesgo de recurrencia ${ }^{10}$. Los inhibidores de la enzima convertidora de angiotensina están indicados si existen disfunción ventricular o hipertensión arterial, y las estatinas en aquellas personas con dislipidemia 0 con aterosclerosis concomitante ${ }^{10}$. Tras el diagnóstico de DCE se recomienda el cribado de DFM y otras lesiones arteriales mediante técnicas de imagen.

El pronóstico de estos pacientes es favorable, presentando una tasa de mortalidad intrahospitalaria y a largo plazo baja. Sin embargo, existe un riesgo significativo de recurrencia ${ }^{6}, y$, generalmente, la disección de novo ocurre en otro territorio coronario. Por este motivo, es importante el seguimiento estrecho tras el evento coronario agudo.

\section{Financiamiento}

Ninguno.

\section{Conflicto de intereses}

Los autores declaran no tener conflicto de intereses.

\section{Responsabilidades éticas}

Protección de personas y animales. Los autores declaran que para esta investigación no se han realizado experimentos en seres humanos ni en animales.

Confidencialidad de los datos. Los autores declaran que han seguido los protocolos de su centro 


\section{de trabajo sobre la publicación de datos de pacientes.}

Derecho a la privacidad y consentimiento informado. Los autores han obtenido el consentimiento informado de los pacientes y/o sujetos referidos en el artículo. Este documento obra en poder del autor de correspondencia.

\section{Bibliografía}

1. Saw J. Spontaneous coronary artery dissection. Can J Cardiol 2013;29:1027-33.

2. Saw J, Mancini GBJ, Humphries KH. Contemporary review on spontaneous coronary artery dissection. J Am Coll Cardiol. 2016;68:297-312.

3. Saw J. Coronary angiogram classification of spontaneous coronary artery dissection. Catheter Cardiovasc Interv. 2014;84:1115-22.
4. Gilhofer TS, Saw J. Spontaneous coronary artery dissection: a review of complications and management strategies. Expert Rev Cardiovasc Ther. 2019;17:275-91.

5. Hassan S, Prakash R, Starovoytov A, Saw J. Natural history of spontaneous coronary artery dissection with spontaneous angiographic healing. JACC Cardiovasc Interv. 2019;12(6):518-27.

6. Tan NY, Tweet MS. Spontaneous coronary artery dissection: etiology and recurrence. Expert Rev Cardiovasc Ther. 2019;17(7):497-510.

7. Hayes SN, Kim ESH, Saw J, Adlam D, Arslanian-Engoren C, Economy KE, et al. Spontaneous coronary artery dissection: current state of the science: a scientific statement from the American Heart Association. Circulation. 2018;137:e523-e557.

8. Hayes SN, Tweet MS, Adlam D, Kim ESH, Gulati R, Price JE, et al. Spontaneous coronary artery dissection: JACC State-of-the-Art Review. J Am Coll Cardiol. 2020;76(8):961-84.

9. Collet JP, Thiele H, Barbato E, Barthélémy O, Bauersachs J, Bhatt DL, et al. 2020 ESC Guidelines for the management of acute coronary syndromes in patients presenting without persistent ST-segment elevation. Eur Heart J. 2021;42(14):1289-367.

10. Saw J, Humphries K, Aymong E, Sedlak T, Prakash R, Starovoytov A, et al. Spontaneous coronary artery dissection: clinical outcomes and risk of recurrence. J Am Coll Cardiol. 2017;70:1148-58. 